Supporting information

\title{
Cross-Linked Chains of Metal-Organic Framework Afford Continuous Ion Transport in Solid Batteries
}

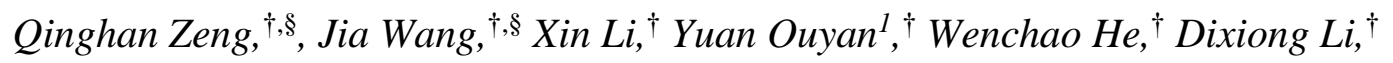

Sijia Guo, ${ }^{\dagger}$ Yingbo Xiao, ${ }^{\dagger}$ Haoyan Deng, ${ }^{\dagger}$ Wei Gong,${ }^{\dagger}$ Qi Zhang ${ }^{*}, \dagger, \dagger$ and Shaoming Huang $*, \dagger$,

†Guangzhou Key Laboratory of Low-Dimensional Materials and Energy Storage

Devices, School of Materials and Energy, Guangdong University of Technology, Guangzhou, 510006, People's Republic of China

${ }^{\ddagger}$ Synergy Innovation Institute of GDUT, Heyuan, 517000, People’s Republic of China

*Email for Q.Z.: qzhangmse@gdut.edu.cn

*Email for S.H.: smhuang@gdut.edu.cn 


\section{Experimental Procedures}

\section{Materials}

All chemical reagents such as Zirconium chloride $\left(\mathrm{ZrCl}_{4}, 99.9 \%\right.$, Sigma-Aldrich), acetic acid (99.5\% Sigma-Aldrich), N,N-dimethylformamide (DMF, 99.9\%, SigmaAldrich), bis(trifluoromethanesulfoneimide) lithium salt (LiTFSI, 99\%, Macklin), bis(trifluoromethane)sulfonimide sodium salt (NaTFSI, 99\%, Macklin), potassium hexafluorophosphate $\left(\mathrm{KPF}_{6}, 99 \%\right.$, Macklin), zinc perchlorate $\left(\mathrm{Zn}\left(\mathrm{ClO}_{4}\right)_{2} \cdot 6 \mathrm{H}_{2} \mathrm{O}, 99 \%\right.$, Macklin), poly(vinylidene fluoride-co-hexafluoropropylene) (PVDF-HFP, Mw = 600000, Solvay), propylene carbonate (PC, 99.9\%, Sigma-Aldrich), N-methyl pyrrolidone (NMP, 99.9\%, Sigma-Aldrich), BC hydrogel (Hainan Guangyu Biotechnology Co., Ltd), BC suspension $(0.55 \mathrm{wt} \%$, Canrd) were used as received without further processing. $\mathrm{H}_{2} \mathrm{BPDC}-2 \mathrm{SO}_{3} \mathrm{H} \quad\left(2^{\prime}, 5^{\prime}\right.$-disulfo-[1, 1':4', 1"-terphenyl]3,3",5,5"-tetracarboxylic acid) was prepared according to a literature procedure. ${ }^{1}$

\section{Synthesis of $\mathrm{Zr}-\mathrm{BPDC}-2 \mathrm{SO}_{3} \mathrm{H}$}

A mixture of $\mathrm{H}_{2} \mathrm{BPDC}-2 \mathrm{SO}_{3} \mathrm{H}(161.25 \mathrm{mg}, 0.43 \mathrm{mmol})$ and $\mathrm{ZrCl}_{4}(99.82 \mathrm{mg}, 0.43$ mmol) was dissolved in a solution of DMF $(12 \mathrm{~mL})$ and acetic acid $(600 \mu \mathrm{L})$. The solution was then transferred into a $20 \mathrm{~mL}$ Teflon-lined autoclave and subjected to ultrasonic treatment for $5 \mathrm{~min}$. Subsequently, the autoclave was kept at $120{ }^{\circ} \mathrm{C}$ for 48 h. The sediment was collected by centrifugation and washed with ethanol for 3 times and dried at $60{ }^{\circ} \mathrm{C}$ for $12 \mathrm{~h}$. Finally, the powder was activated by heating at $150{ }^{\circ} \mathrm{C}$ in vacuum. 


\section{Synthesis of $\mathrm{Zr}-\mathrm{BPDC}-2 \mathrm{SO}_{3} \mathrm{M}$}

Zr-BPDC-2SO $\mathrm{SO}_{3} \mathrm{H}$ was placed in a prepared solution of $1 \mathrm{M}$ LiTFSI, $1 \mathrm{M}$ NaTFSI, $1 \mathrm{M}$

$\mathrm{KPF}_{6}$ and $1 \mathrm{M} \mathrm{Zn}\left(\mathrm{ClO}_{4}\right)_{2} \cdot 6 \mathrm{H}_{2} \mathrm{O}$ in $\mathrm{PC}$, respectively. The mixture was then kept in a shaker for 6 hours to adsorb metal cations. Then the solids were obtained by centrifugation and washed with PC and dried at $100{ }^{\circ} \mathrm{C}$. The obtained MOFs are named as $\mathrm{Zr}-\mathrm{BPDC}-2 \mathrm{SO}_{3} \mathrm{M}\left(\mathrm{M}=\mathrm{Li}^{+}, \mathrm{Na}^{+}, \mathrm{K}^{+}\right.$, and $\left.\mathrm{Zn}^{2+}\right)$. For the XPS test, $\mathrm{Zr}-\mathrm{BPDC}-2 \mathrm{SO}_{3} \mathrm{Li}$ was obtained by using acetonitrile as the solvent instead and the final sample was dried at $150{ }^{\circ} \mathrm{C}$ under vacuum for $12 \mathrm{~h}$.

\section{Synthesis of Zr-BPDC-2SO3 $\mathrm{Li}^{+} / \mathrm{PVDF}-\mathrm{HFP}$ membrane}

Zr-BPDC-2SO $\mathrm{Li}^{+}(400 \mathrm{mg})$, PVDF-HFP $(100 \mathrm{mg})$ and LiTFSI $(57 \mathrm{mg})$ were mixed in DMF. After magnetically stirring for $24 \mathrm{~h}$, the mixture was casted into cleaned petri dishes and prebaked for half an hour under infrared lamp. Then the mixture was further dried at $80{ }^{\circ} \mathrm{C}$ under vacuum for $24 \mathrm{~h}$ to remove the DMF solvent. The obtained flexible membranes were stored in an argon filled glove box $\left(\leq 0.01 \mathrm{ppm} \mathrm{H}_{2} \mathrm{O}\right.$ and $\left.\mathrm{O}_{2}\right)$ (MIKROUNA).

\section{Synthesis of $\mathrm{Zr}-\mathrm{BPDC}-2 \mathrm{SO}_{3} \mathrm{H} / \mathrm{BC}$ membrane}

$70 \mathrm{mg}$ of $\mathrm{Zr}-\mathrm{BPDC}-2 \mathrm{SO}_{3} \mathrm{H}$ was dispersed in $5.45 \mathrm{~g}$ of $\mathrm{BC}$ suspension $(0.55 \mathrm{wt} \%$ in water) with $10 \mathrm{~mL}$ ethanol added. The mixture was stirred vigorously for $5 \mathrm{~h}$. Finally, the membrane was obtained by vacuum filtration and washed with ethanol for several times. After drying at $80{ }^{\circ} \mathrm{C}$ under vacuum for $24 \mathrm{~h}$, the free-standing $\mathrm{Zr}$-BPDC$2 \mathrm{SO}_{3} \mathrm{H} / \mathrm{BC}$ membrane can be easily peeled off from the filter membrane. The obtained 
flexible membranes were cut into disks with a diameter of $16 \mathrm{~mm}$ and stored in an argon filled glove box ( $\leq 0.01 \mathrm{ppm} \mathrm{H}_{2} \mathrm{O}$ and $\mathrm{O}_{2}$ ) (MIKROUNA).

\section{Synthesis of the cross-linked MOF chains membrane}

The membrane consisted of $\mathrm{BC}$ nanofibers can be obtained by freeze-drying $\mathrm{BC}$ hydrogel for $48 \mathrm{~h}$ and was further cut into disks with a diameter of $16 \mathrm{~mm}$. Then the BC membranes were immersed in $\mathrm{ZrCl}_{4}$ solution in DMF under ultrasonic treatment at room temperature for about $20 \mathrm{~min}$. The corresponding organic ligand and acetic acid were then added. The amounts of the chemicals were the same as the synthesis of $\mathrm{Zr}$ BPDC-2 $\mathrm{SO}_{3} \mathrm{H}$. After being dispersed under ultrasonic treatment for $5 \mathrm{~min}$, the mixture was transferred into a Teflon-lined autoclave and heated at $120{ }^{\circ} \mathrm{C}$ for $48 \mathrm{~h}$. The yielded membranes were washed by ethanol for several times and dried at $80^{\circ} \mathrm{C}$ under vacuum to obtain the white cross-linked MOF chains membranes. The obtained membranes

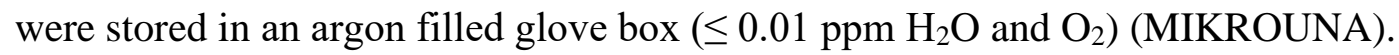

\section{Preparation of cathode}

Commercial cathode material $\left(\mathrm{LiFePO}_{4}\right.$ or $\left.\mathrm{LiCoO}_{2}\right)$, super-P and PVDF were mixed in $\mathrm{N}$-methyl pyrrolidone (NMP) with a mass ratio of 8:1:1 to obtain the cathode mixture. And the cathode mixture was subsequently coated on an aluminium foil. The prepared electrode films were dried at $60{ }^{\circ} \mathrm{C}$ for $24 \mathrm{~h}$ under vacuum before cell fabrication. The electrode films were punched into disks with a diameter of $12 \mathrm{~mm}$. The loading of the active material is around $2.3 \mathrm{mg} \mathrm{cm}{ }^{-2}$ and $3.5 \mathrm{mg} \mathrm{cm}^{-2}$ for $\mathrm{LiFePO}_{4}$ and $\mathrm{LiCoO}_{2}$, respectively. 
For the $\mathrm{LiFePO}_{4}$ cathode with high active material loading, commercial $\mathrm{LiFePO}_{4}$, super-P and PVDF were mixed in NMP with a mass ratio of 9:0.5:0.5 to obtain the cathode mixture. The cathode mixture was subsequently coated on an aluminium foil by using the coater. The prepared electrode films were dried at $80^{\circ} \mathrm{C}$ for $12 \mathrm{~h}$ and then $120^{\circ} \mathrm{C}$ for $4 \mathrm{~h}$ under vacuum before cell fabrication. The electrode films were punched into disks with a diameter of $12 \mathrm{~mm}$. The loading of $\mathrm{LiFePO}_{4}$ is around $14.6 \mathrm{mg} \mathrm{cm}^{-2}$.

\section{Characterization}

The SEM images were captured using FEI Apreo C. Powder X-ray diffraction (PXRD) patterns were collected with a SmartLabTM $9 \mathrm{~kW}$ X-ray diffractometer $(\mathrm{Cu} \mathrm{K \alpha}$ radiation, $\lambda=1.541 \AA ; 2 \theta$ from 3 to $30^{\circ}$ ). Thermogravimetric analyses (TGA) were performed with a Perkin Elmer UNIX/TGA7 system under nitrogen $\left(\mathrm{N}_{2}\right)$ atmosphere from room temperature to $250{ }^{\circ} \mathrm{C}$ at a heating rate of $10{ }^{\circ} \mathrm{C} \mathrm{min}^{-1}$ and then kept at $250{ }^{\circ} \mathrm{C}$ for one hour. The specific surface area was obtained by the Brunauer Emmett Teller method using nitrogen adsorption (Micromeritics ASAP 2460). The X-ray photoelectron spectroscopy (XPS) spectra were collected with Thermo Fisher Escalab 250Xi analyzer. Contents of metal ions in the samples were detected by inductively coupled plasma atomic emission spectrometry(ICP-AES) on a Thermo Fisher ICAP RQ. Ionic conductivity, interfacial compatibilities, lithium-ion transference number $\left(t_{\mathrm{Li}^{+}}{ }^{+}\right.$ and electrochemical window were tested with electrochemical workstation (AutoLab 302N). The ionic conductivities were determined using (electrochemical impedance spectroscopy) EIS with the membrane sandwiched between two stainless steel (SS) 
electrodes in a 2032-type coin cell. The frequency range was from $1 \times 10^{6}$ to $0.1 \mathrm{~Hz}$ and the AC amplitude was $10 \mathrm{mV}$. The ionic conductivity was obtained by the Eq. (1)

$$
\sigma=\frac{L}{R \times S}(1)
$$

$L(\mathrm{~cm})$ is the thickness of membranes, $S\left(\mathrm{~cm}^{-2}\right)$ is the area of the electrolyte and $R(\Omega)$ is the bulk resistance.

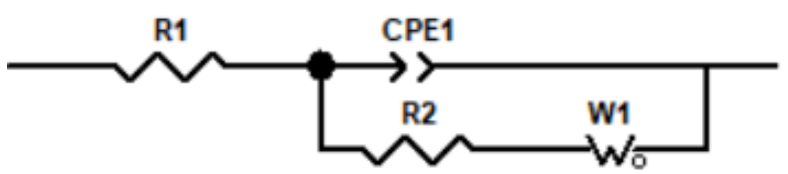

Scheme S1. The equivalent circuit used for fitting impedance spectra for the evaluation of ionic conductivity. $\mathrm{R} 1$ and $\mathrm{R} 2$ are resistors, CPE1 is constant phase element which is an imperfect capacitor and W1 is Warburg element.

Additionally, the interfacial compatibilities were evaluated by testing the interfacial impedance of the $\mathrm{Li}|\mathrm{SE}| \mathrm{Li}$ cells at room temperature $(\mathrm{RT})$ with the frequency ranged from $1 \times 10^{5}$ to $0.1 \mathrm{~Hz}$. Lithium-ion transference number $\left(t_{\mathrm{Li}}{ }^{+}\right)$was measured by combining an AC impedance measurement and a potentiostatic polarization measurement with $\mathrm{Li}|\mathrm{SE}| \mathrm{Li}$ cells with the frequency ranged from $1 \times 10^{5}$ to $0.1 \mathrm{~Hz}$.

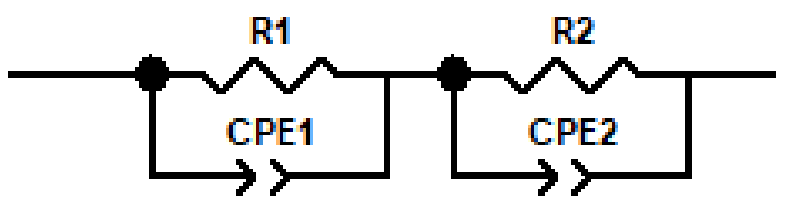


Scheme S2. The equivalent circuit used for fitting impedance spectra for the evaluation of interfacial impedance. R1 and R2 are resistors and CPE1 and CPE2 are constant phase elements which are imperfect capacitors.

$t_{\mathrm{Li}}{ }^{+}$was calculated by the Eq. (2), $\Delta V$ is the dc polarization voltage $(10 \mathrm{mV}), I_{0}$ and $I_{s}$ are initial and stable currents during polarization, $R_{0}$ and $R_{S}$ are the impedances before and after polarization.

$$
t_{L i+}=\frac{I_{S}\left(\Delta V-I_{0} R_{0}\right)}{I_{0}\left(\Delta V-I_{S} R_{S}\right)}
$$

Electrochemical window was evaluated by linear sweep voltammetry (LSV) using Li|SE|SS cells with a scan rate of $1 \mathrm{mV} \mathrm{s}^{-1}$ over the potential range of $0-6 \mathrm{~V}$.

Galvanostatic cycling test was carried out with symmetric Li cells using a Neware CT4008 battery tester under constant current density of $0.1,0.2,0.5,1$ and $2 \mathrm{mAh} \mathrm{cm}^{-2}$ at room temperature, respectively. Critical current density (CCD) was defined as the current density at which the cell was short circuited. The applied current density was increased from 0.1 to $1.5 \mathrm{~mA} \mathrm{~cm}^{-2}$ with a step increase of $0.1 \mathrm{~mA} \mathrm{~cm}^{-2}$ for $1 \mathrm{~h}$ stripping and $1 \mathrm{~h}$ plating at room temperature. The battery performance of $\mathrm{LiFePO}_{4} / \mathrm{Li}$ and $\mathrm{LiCoO}_{2} / \mathrm{Li}$ cells was tested with the voltage range from 2.6 to $4.0 \mathrm{~V}$ and 3 to $4.5 \mathrm{~V}$, respectively. 


\section{Results and Discussion}

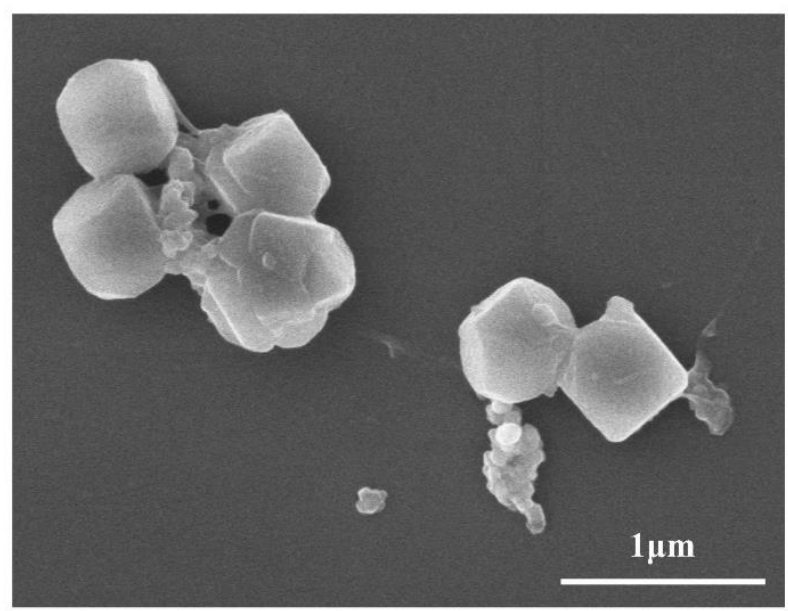

Figure S1. SEM image of synthesized Zr-BPDC-2SO${ }_{3} \mathrm{H}$.
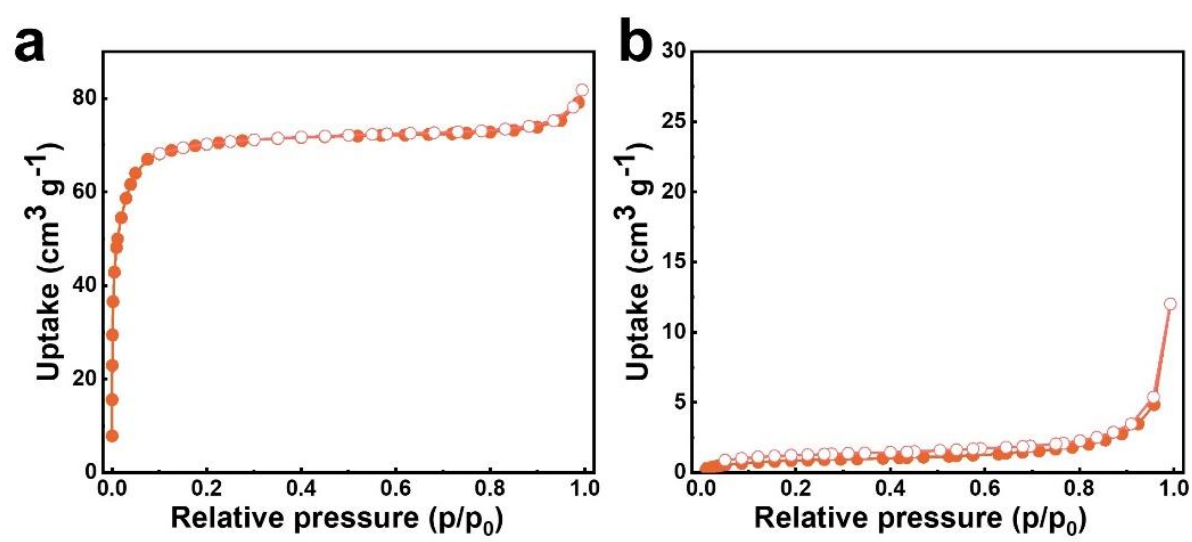

Figure S2. $\mathrm{N}_{2}$ adsorption (filled symbols) and desorption (empty symbols) isotherms at $77 \mathrm{~K}$ for (a) $\mathrm{Zr}-\mathrm{BPDC}-2 \mathrm{SO}_{3} \mathrm{H}$ and (b) $\mathrm{Zr}-\mathrm{BPDC}-2 \mathrm{SO}_{3} \mathrm{Li}$. 

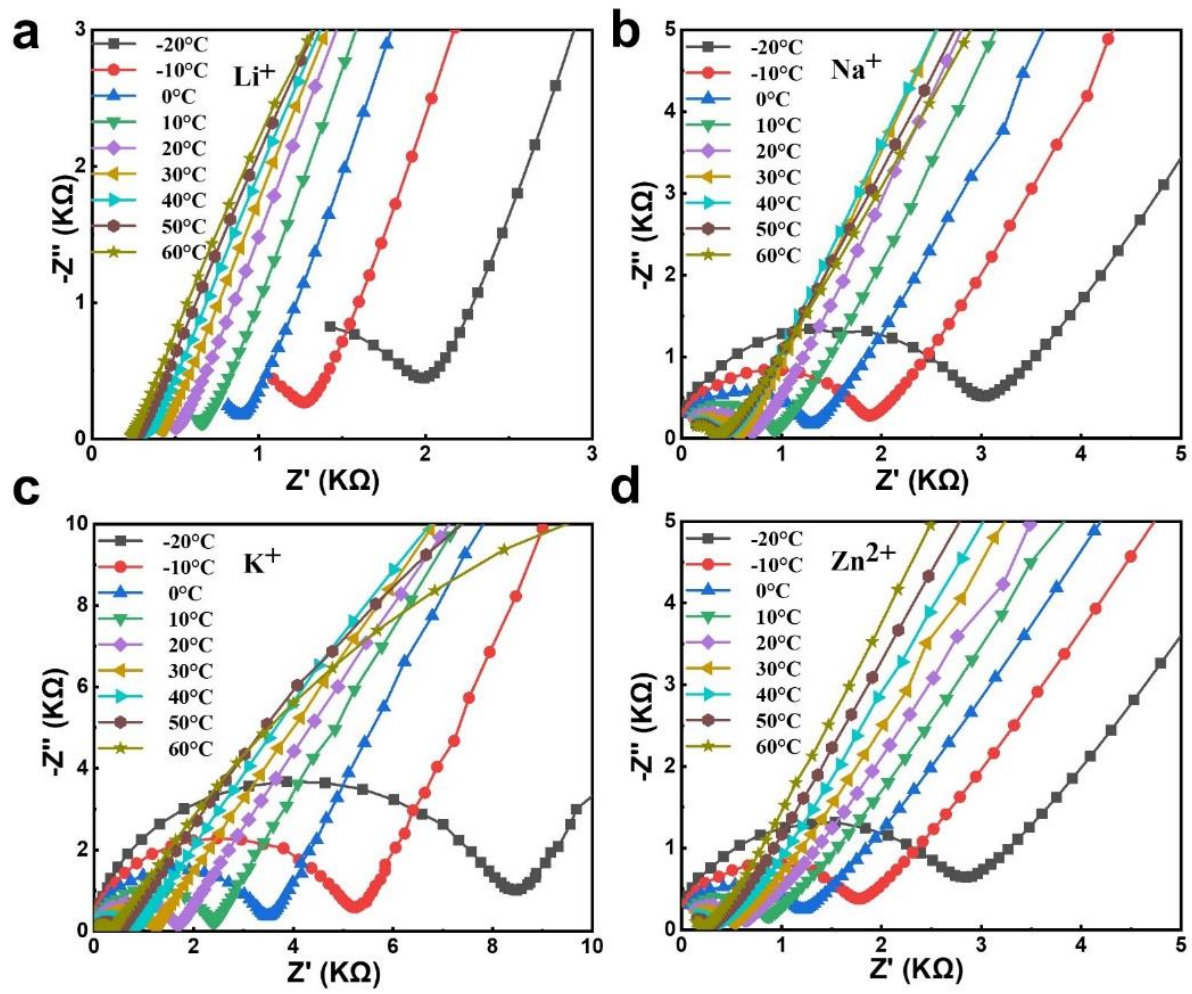

Figure S3. EIS plots of Zr-BPDC-2SO $\mathrm{SO}_{3} \mathrm{H}$ for $\mathrm{Li}^{+}, \mathrm{Na}^{+}, \mathrm{K}^{+}, \mathrm{Zn}^{2+}$ from -20 to $60{ }^{\circ} \mathrm{C}$.

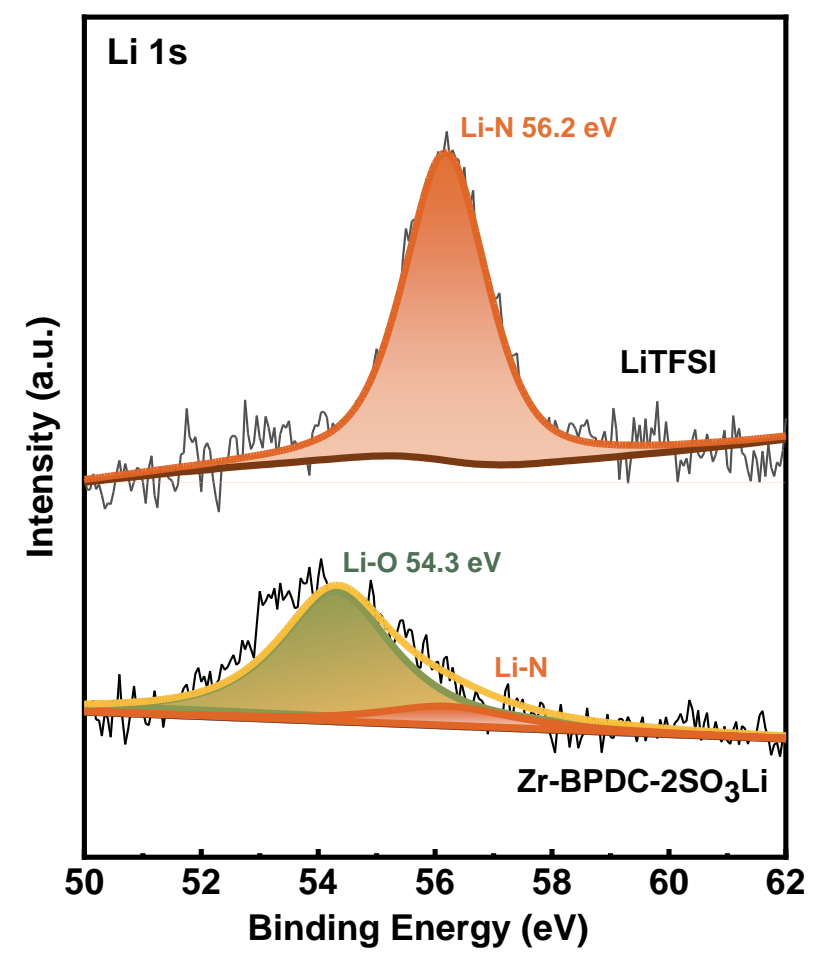

Figure S4. High-resolution of $\mathrm{Li} 1$ s of LiTFSI and $\mathrm{Zr}-\mathrm{BPDC}-2 \mathrm{SO}_{3} \mathrm{Li}$. 

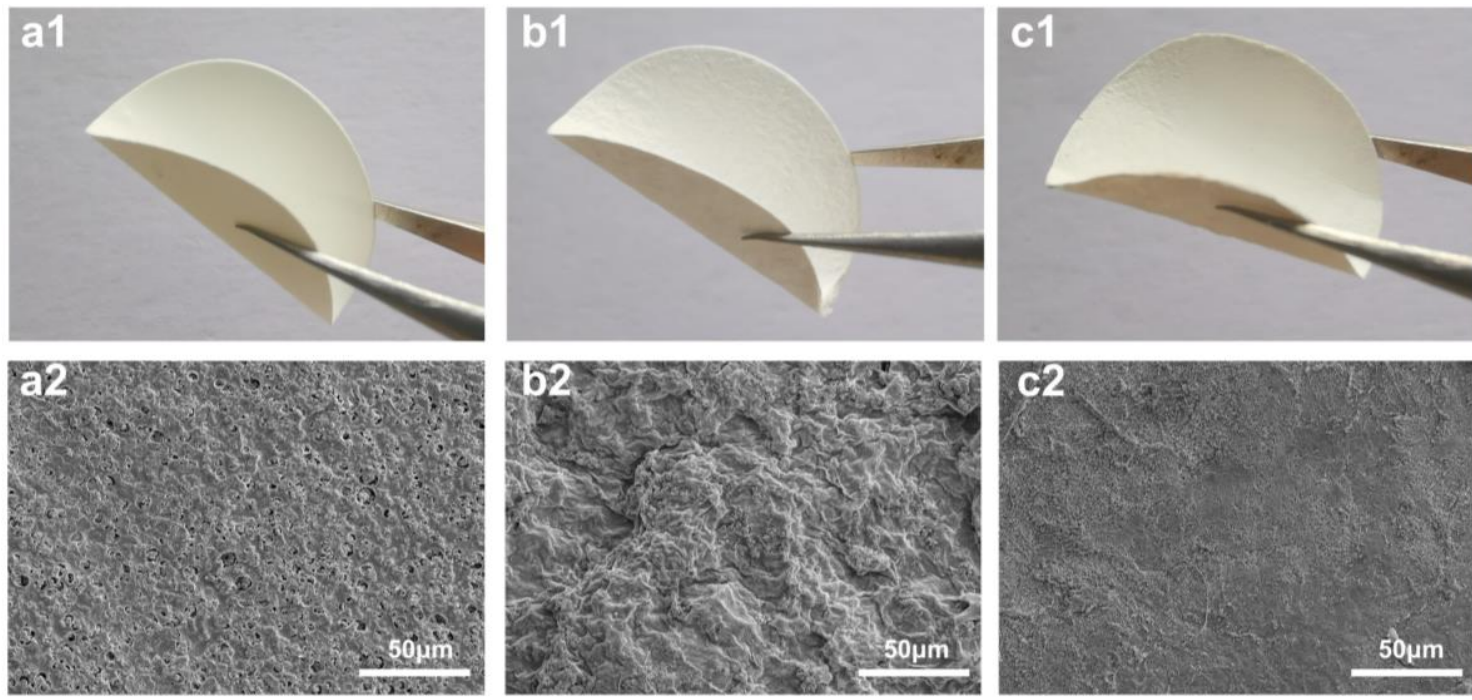

Figure S5. Photographs and SEM images of Zr-BPDC-2SO $\mathrm{Li}^{+} / \mathrm{PVDF}-\mathrm{HFP}(\mathrm{a} 1, \mathrm{a} 2)$, $\mathrm{Zr}-\mathrm{BPDC}-2 \mathrm{SO}_{3} \mathrm{H} / \mathrm{BC}(\mathrm{b} 1, \mathrm{~b} 2)$ and cross-linked MOF chains (c1, c2).

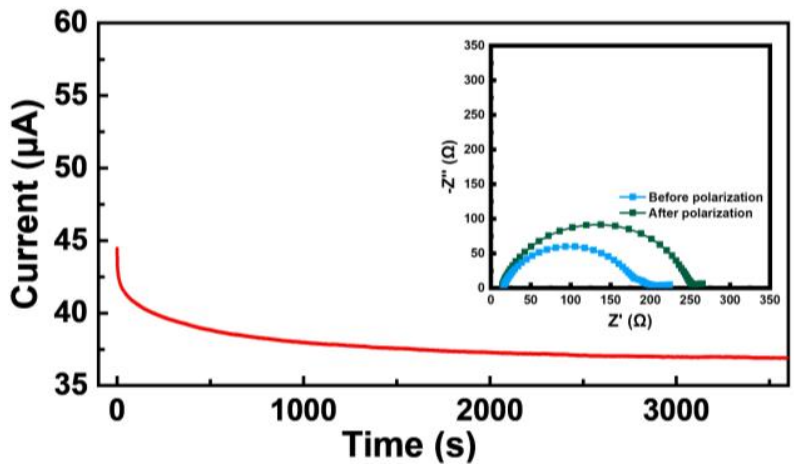

Figure S6. Current-time curve of polarization of the $\mathrm{Li} \mid \mathrm{Zr}-\mathrm{BPDC}-2 \mathrm{SO}_{3} \mathrm{Li}^{+} / \mathrm{PVDF}-$ $\mathrm{HFP} \mid \mathrm{Li}$ cell at $10 \mathrm{mV}$, the inset is the EIS before and after polarization.
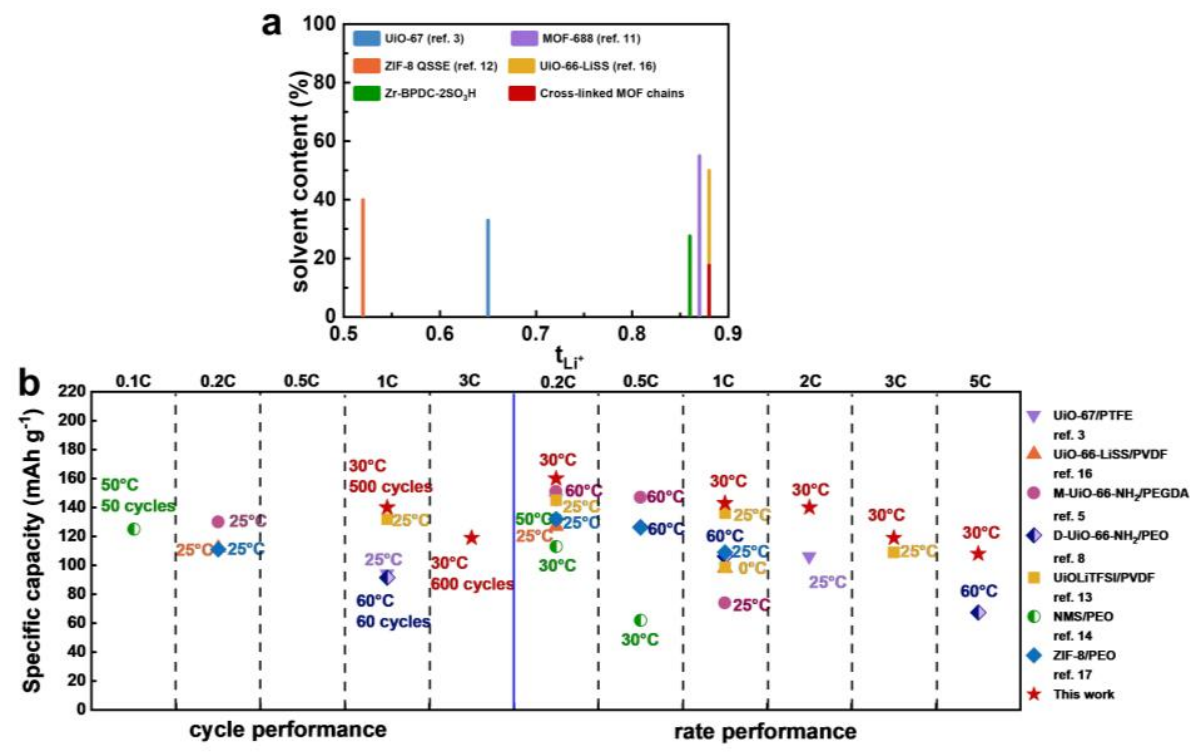
Figure S7. (a) Comparison of $\mathrm{Li}^{+}$transference number and solvent content of previous reported MOF-based SEs. (b) Comparison of electrochemical performance of $\mathrm{LiFePO}_{4} / \mathrm{Li}$ cells in previous reported SSBs.

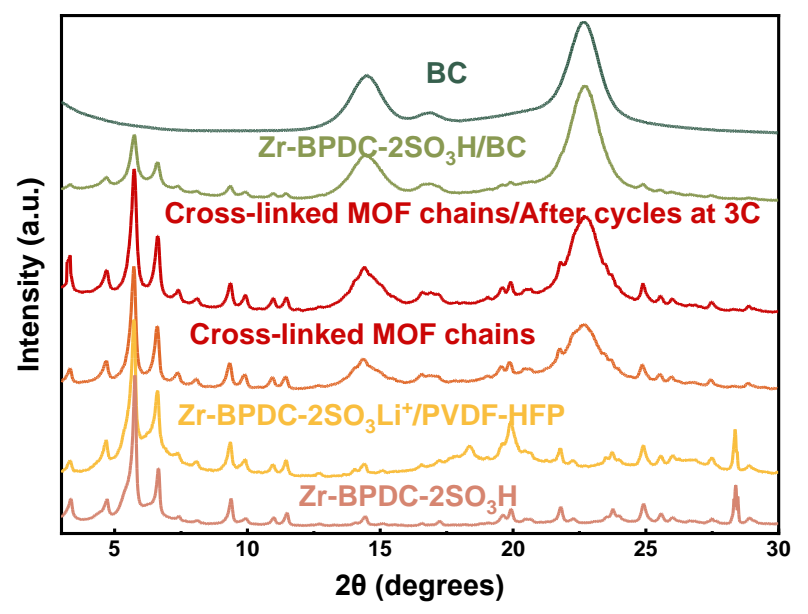

Figure S8. PXRD of Zr-BPDC-2SO ${ }_{3} \mathrm{H}$ nanoparticles, $\mathrm{Zr}-\mathrm{BPDC}-2 \mathrm{SO}_{3} \mathrm{Li}^{+} / \mathrm{PVDF}-\mathrm{HFP}$, cross-linked MOF chains, cross-linked MOF chains after cycles at 3C, Zr-BPDC$2 \mathrm{SO}_{3} \mathrm{H} / \mathrm{BC}$ and $\mathrm{BC}$.

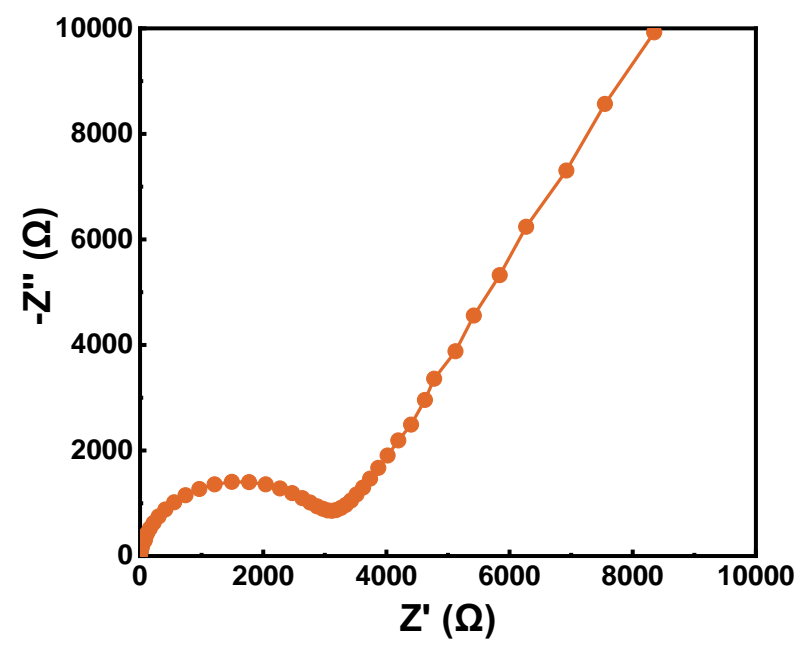

Figure S9. EIS plots of cross-linked MOF chains with $\mathrm{Li}^{+}$with the solvent completely removed. 

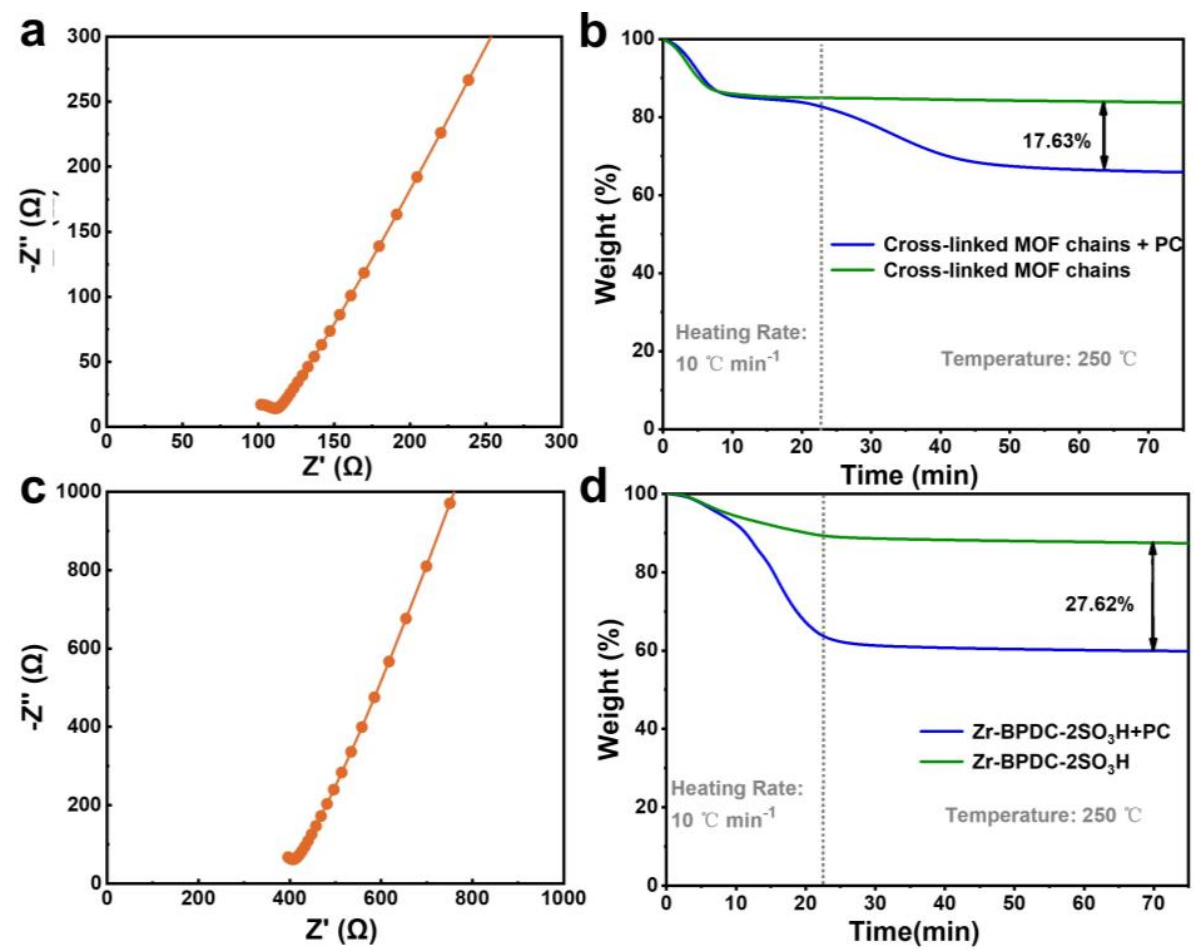

Figure S10. (a) EIS plots and (b)TGA plots of cross-linked MOF chains with $\mathrm{Li}^{+}$from room temperature to $250{ }^{\circ} \mathrm{C}$ at a heating rate of $10{ }^{\circ} \mathrm{C} \min ^{-1}$ in $\mathrm{N}_{2}$ atmosphere. (c) EIS plots and (d)TGA plots of $\mathrm{Zr}$-BPDC-2SO $3 \mathrm{H}^{\mathrm{H}}$ with $\mathrm{Li}^{+}$from room temperature to $250{ }^{\circ} \mathrm{C}$ at a heating rate of $10{ }^{\circ} \mathrm{C} \min ^{-1}$ in $\mathrm{N}_{2}$ atmosphere.
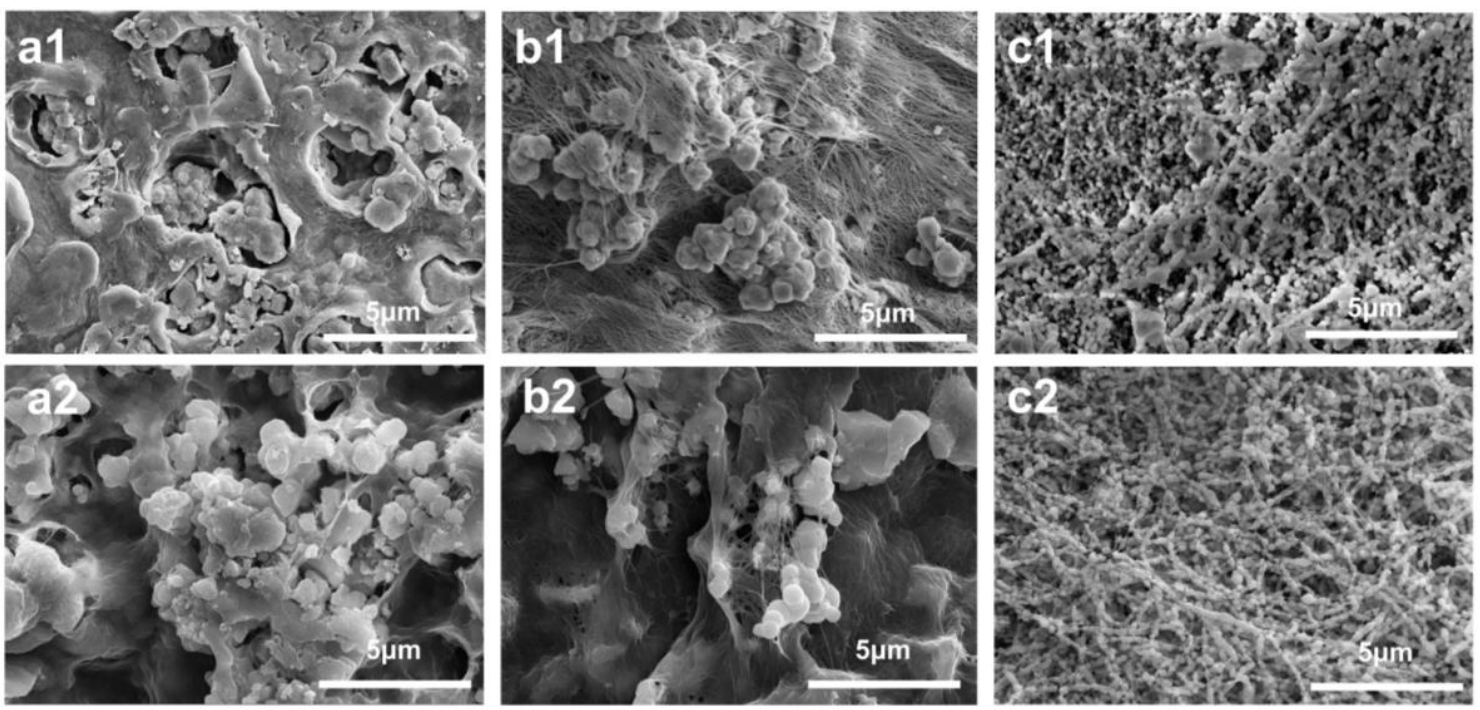

Figure S11. SEM images of (a1) Zr-BPDC-2SO${ }_{3} \mathrm{Li}^{+} / \mathrm{PVDF}-\mathrm{HFP}$ and (a2) Zr-BPDC$2 \mathrm{SO}_{3} \mathrm{Li}^{+} / \mathrm{PVDF}-\mathrm{HFP}$ after 50 cycles at $1 \mathrm{C}$, (b1) Zr-BPDC-2SO ${ }_{3} \mathrm{H} / \mathrm{BC}$ and (b2) ZrBPDC-2 $\mathrm{SO}_{3} \mathrm{H} / \mathrm{BC}$ after 50 cycles at $1 \mathrm{C},(\mathrm{c} 1)$ cross-linked MOF chains and (c2) crosslinked MOF chains after 50 cycles at $1 \mathrm{C}$. 

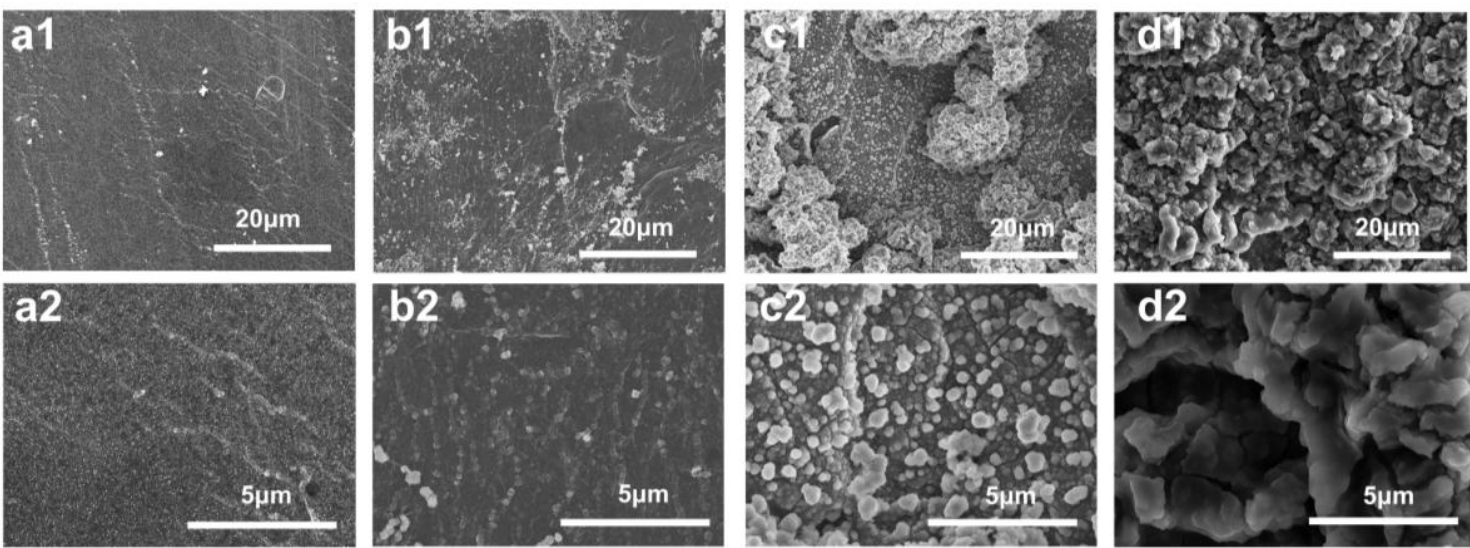

Figure S12. Surface morphologies of lithium plates before (a1, a2) and after 50 cycles of plating/stripping tests with (b1, b2) cross-linked MOF chains, (c1, c2) Zr-BPDC$2 \mathrm{SO}_{3} \mathrm{Li}^{+} / \mathrm{PVDF}-\mathrm{HFP}$ and $(\mathrm{d} 1, \mathrm{~d} 2) \mathrm{Zr}-\mathrm{BPDC}-2 \mathrm{SO}_{3} \mathrm{H} / \mathrm{BC}$ as SE in SSB.

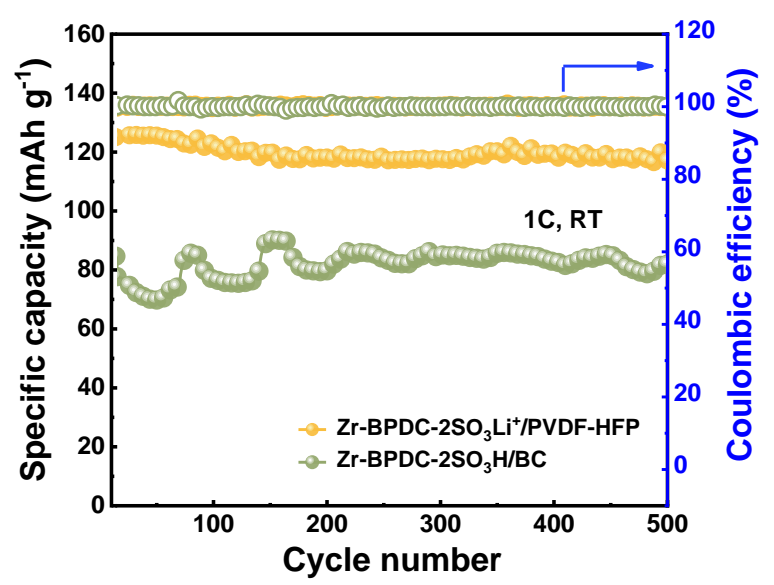

Figure S13. Cycling performance of $\mathrm{LiFePO}_{4} / \mathrm{Li}$ fabricated with $\mathrm{Zr}$-BPDC$2 \mathrm{SO}_{3} \mathrm{Li}^{+} / \mathrm{PVDF}-\mathrm{HFP}$ and $\mathrm{Zr}-\mathrm{BPDC}-2 \mathrm{SO}_{3} \mathrm{H} / \mathrm{BC}$ at $1 \mathrm{C}$.
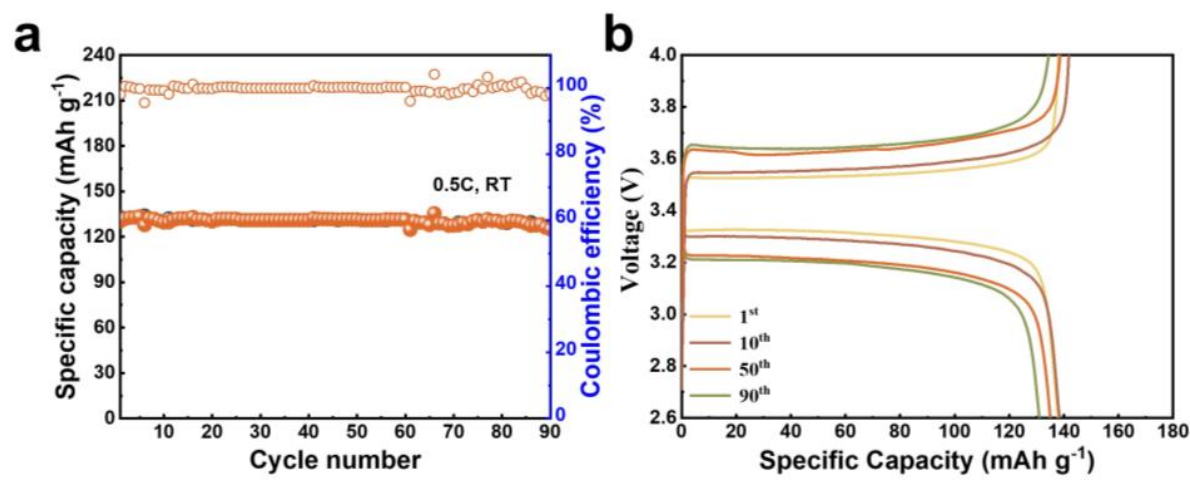

Figure S14. (a-b) Cycling performance and charge/discharge profiles of Li|cross-linked MOF chains $\mid \mathrm{LiFePO}_{4}$ cell at $0.5 \mathrm{C}$ under room temperature with high active material loadings. 

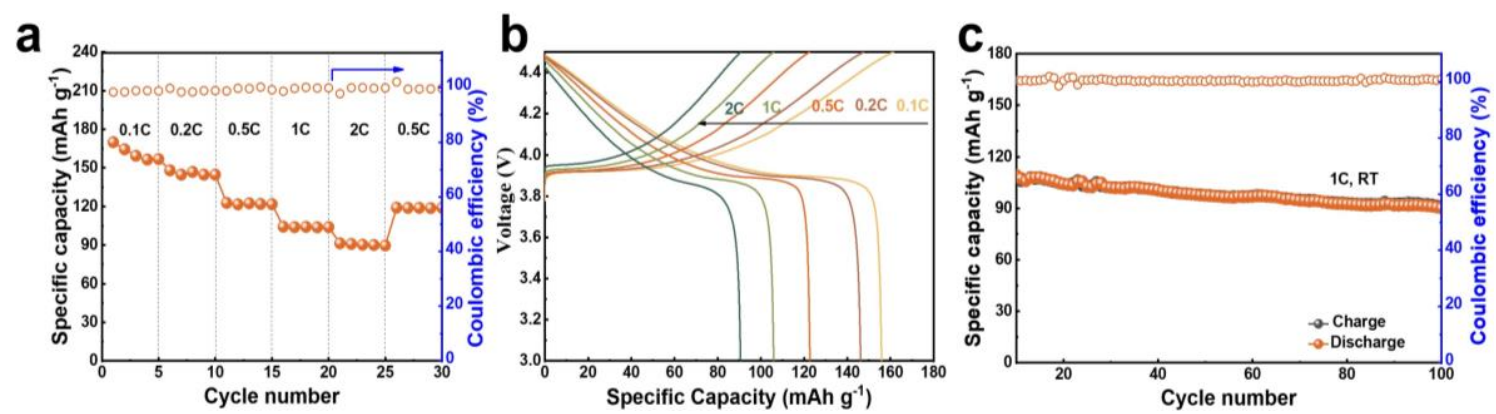

Figure S15. (a-b) C-rate performance and charge/discharge profiles of a Li|cross-linked MOF chains $\mid \mathrm{LiCoO}_{2}$ cell under room temperature. (c) Cycling performance of Li|crosslinked $\mathrm{MOF}$ chains $\mid \mathrm{LiCoO}_{2}$ cell at $1 \mathrm{C}$ under room temperature.

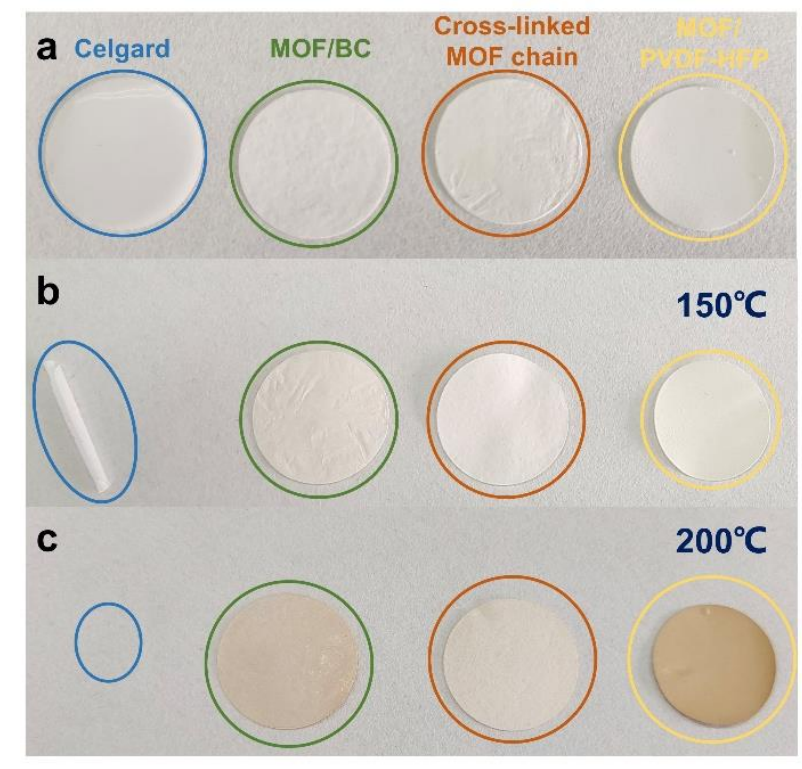

Figure S16. (a-c) Photographs of Celgard separator, Zr-BPDC-2 $\mathrm{SO}_{3} \mathrm{Li}^{+} / \mathrm{PVDF}-\mathrm{HFP}$, $\mathrm{Zr}-\mathrm{BPDC}-2 \mathrm{SO}_{3} \mathrm{H} / \mathrm{BC}$ and cross-linked $\mathrm{MOF}$ chains after heated at $30{ }^{\circ} \mathrm{C}, 150{ }^{\circ} \mathrm{C}$ and $200{ }^{\circ} \mathrm{C}$, respectively. 
Table S1. Comparison of the ion conductivity and the $\mathrm{Li}^{+}$transference number of previous reported MOF-based SEs. (RT: Room Temperature)

\begin{tabular}{|c|c|c|c|c|c|}
\hline Materials & $\mathrm{t}_{\mathrm{Li}}^{+}$ & $\sigma\left(\mathrm{S} \mathrm{cm}^{-1}\right)$ & Description & content & Ref. \\
\hline $\begin{array}{c}\mathrm{Mg}_{2}(\text { dobdc }) \\
0.35 \mathrm{LiO}^{\mathrm{i}} \mathrm{Pr} \cdot 0 . \\
25 \mathrm{LiBF}_{4}\end{array}$ & - & $3.1 \times 10^{-4}\left(27^{\circ} \mathrm{C}\right)$ & $\mathrm{MOF}+\mathrm{EC} \cdot \mathrm{DEC}$ & - & 2 \\
\hline MIT-20-LiCl & 0.66 & $1.3 \times 10^{-5}\left(25^{\circ} \mathrm{C}\right)$ & $\mathrm{MOF}+\mathrm{PC}$ & $\begin{array}{c}10.3 \text { per } \\
\text { mole of } \\
\text { MOF }\end{array}$ & 3 \\
\hline UiO-67 & 0.65 & $6.5 \times 10^{-4}(\mathrm{RT})$ & $\mathrm{MOF}+\mathrm{PC}$ & $33 \%$ & 4 \\
\hline $\begin{array}{l}\text { MOF-525 } \\
\quad(\mathrm{Cu})\end{array}$ & 0.36 & $3.0 \times 10^{-4}(\mathrm{RT})$ & MOF+Li-IL & - & 5 \\
\hline HSPE-1-8 & - & $4.3 \times 10^{-5}\left(30^{\circ} \mathrm{C}\right)$ & $\begin{array}{l}\text { MOF+PEGDA } \\
+\mathrm{LiTFSI}+\mathrm{THF}\end{array}$ & - & 6 \\
\hline $\mathrm{UiO}-67$ & - & $10^{-4}(\mathrm{RT})$ & $\begin{array}{c}\mathrm{MOF}+\mathrm{LLZO}+ \\
\mathrm{Li}-\mathrm{IL}\end{array}$ & - & 7 \\
\hline ZIF-67 & - & $2.29 \times 10^{-3}\left(30^{\circ} \mathrm{C}\right)$ & MOF+IL & - & 8 \\
\hline $\begin{array}{l}\text { D-UiO-66- } \\
\quad \mathrm{NH}_{2}\end{array}$ & 0.72 & $3.1 \times 10^{-5}(\mathrm{RT})$ & $\begin{array}{c}\mathrm{MOF}+\mathrm{PEO}+\mathrm{LiT} \\
\text { FSI }\end{array}$ & $\begin{array}{l}\text { Without } \\
\text { organic } \\
\text { solvent }\end{array}$ & 9 \\
\hline $\begin{array}{c}\mathrm{Cu}_{4}(\mathrm{ttpm})_{2}(\mathrm{C} \\
\left.\mathrm{uCl}_{2}\right)_{0.6}(\mathrm{LiCl}) \\
1.8\end{array}$ & 0.69 & $2.4 \times 10^{-5}\left(25^{\circ} \mathrm{C}\right)$ & $\mathrm{MOF}+\mathrm{PC}$ & $\begin{array}{c}19 \text { per mole } \\
\text { of } \mathrm{MOF}\end{array}$ & 10 \\
\hline PEO-n-UiO & 0.26 & $1.3 \times 10^{-4}\left(30^{\circ} \mathrm{C}\right)$ & $\begin{array}{c}\mathrm{MOF}+\mathrm{PEO}+\mathrm{Li}- \\
\mathrm{IL}\end{array}$ & - & 11 \\
\hline MOF-688 & 0.87 & $3.4 \times 10^{-4}\left(20^{\circ} \mathrm{C}\right)$ & $\mathrm{MOF}+\mathrm{PC}$ & $55 \%$ & 12 \\
\hline ZIF-8 QSSE & 0.52 & $10^{-4}\left(25^{\circ} \mathrm{C}\right)$ & $\begin{array}{c}\mathrm{MOF}+\mathrm{PTFE} \\
+\mathrm{EC} \\
+\mathrm{DMC}+\mathrm{EMC}\end{array}$ & $40 \%$ & 13 \\
\hline UiO-66- $\mathrm{NH}_{2}$ & 0.84 & $2.07 \times 10^{-4}(\mathrm{RT})$ & MOF+LiTFSI & - & 14 \\
\hline NMS & $\begin{array}{c}0.37 \\
8\end{array}$ & $1.66 \times 10^{-5}(\mathrm{RT})$ & $\begin{array}{c}\text { MOF+LiTFSI+ } \\
\text { PEO }\end{array}$ & - & 15 \\
\hline
\end{tabular}




\begin{tabular}{|c|c|c|c|c|c|}
\hline $\begin{array}{c}\text { UiO-66- } \\
\mathrm{NH}_{2} @ \mathrm{SiO}_{2}\end{array}$ & 0.68 & $8.1 \times 10^{-5}\left(60^{\circ} \mathrm{C}\right)$ & $\begin{array}{c}\text { MOF+LiTFSI+ } \\
\text { PEO }\end{array}$ & - & 16 \\
\hline UiO-66-LiSS & 0.88 & $7.8 \times 10^{-4}(\mathrm{RT})$ & $\mathrm{MOF}+\mathrm{PC}+\mathrm{EC}$ & $50 \%$ & 17 \\
\hline $\begin{array}{c}\text { PEO- } \\
\text { LiTFSI/ZIF-8 }\end{array}$ & 0.36 & $2.2 \times 10^{-5}\left(30^{\circ} \mathrm{C}\right)$ & $\mathrm{MOF}+\mathrm{PEO}$ & - & 18 \\
\hline $\begin{array}{c}\text { Li-IL@UiO- } \\
67\end{array}$ & 0.45 & $4.3 \times 10^{-4}(\mathrm{RT})$ & MOF+IL & - & 19 \\
\hline LCMOF & 0.58 & $1.06 \times 10^{-3}(\mathrm{RT})$ & $\begin{array}{c}\mathrm{MOF}+\mathrm{PVDF}+\mathrm{I} \\
\mathrm{L}\end{array}$ & - & 20 \\
\hline $\begin{array}{c}\text { Li- } \\
\text { IL@HKUST- } \\
1\end{array}$ & 0.13 & $1.2 \times 10^{-4}\left(30^{\circ} \mathrm{C}\right)$ & MOF+PEO+IL & - & 21 \\
\hline ZIF-8 & 0.67 & $4.26 \times 10^{-4}\left(30^{\circ} \mathrm{C}\right)$ & $\mathrm{MOF}+\mathrm{PEO}+\mathrm{IL}$ & - & 22 \\
\hline $\begin{array}{l}\text { SPE2-PI- } \\
\text { ZIF8 }\end{array}$ & 0.68 & $4.7 \times 10^{-4}\left(25^{\circ} \mathrm{C}\right)$ & $\begin{array}{c}\mathrm{MOF}+\mathrm{PEO}+\mathrm{PE} \\
\mathrm{DGE}+\mathrm{EC} / \mathrm{DEC}\end{array}$ & $60 \%$ & 24 \\
\hline PMLSE & 0.62 & $2 \times 10^{-4}\left(25^{\circ} \mathrm{C}\right)$ & $\begin{array}{c}\mathrm{PEO}+\mathrm{MOF}+ \\
\mathrm{LLZN+EC/DEC}\end{array}$ & $\begin{array}{c}1.50 \mu \mathrm{L} \\
\text { electrolyte }\end{array}$ & 25 \\
\hline $\begin{array}{c}\mathrm{Zr}-\mathrm{BPDC}- \\
2 \mathrm{SO}_{3} \mathrm{H}\end{array}$ & 0.86 & $2.65 \times 10^{-4}(\mathrm{RT})$ & $\mathrm{MOF}+\mathrm{PC}$ & $27.62 \%$ & $\begin{array}{l}\text { This } \\
\text { work }\end{array}$ \\
\hline $\begin{array}{l}\text { Cross-linked } \\
\text { MOF chains }\end{array}$ & 0.88 & $7.88 \times 10^{-4}(\mathrm{RT})$ & $\mathrm{MOF}+\mathrm{BC}+\mathrm{PC}$ & $17.63 \%$ & $\begin{array}{l}\text { This } \\
\text { work }\end{array}$ \\
\hline
\end{tabular}


Table S2. Comparison of electrochemical performance of $\mathrm{LiFePO}_{4} / \mathrm{Li}$ cells in previous reported SSBs.

\begin{tabular}{|c|c|c|c|c|c|}
\hline Component & $\begin{array}{l}\text { Mixing } \\
\text { type }\end{array}$ & $\begin{array}{c}\text { Interface } \\
\text { impedan } \\
\text { ce }\end{array}$ & $\begin{array}{c}\text { Cycle } \\
\text { performance } \\
\left(\mathrm{mAh} \mathrm{g}^{-1}\right)\end{array}$ & $\begin{array}{l}\text { Rate performance } \\
\qquad\left(\mathrm{mAh}^{-1}\right)\end{array}$ & $\begin{array}{c}\text { Ref } \\
.\end{array}$ \\
\hline $\begin{array}{c}\text { UiO-67/PTFE } \\
(9: 1)\end{array}$ & $\begin{array}{l}\text { Physical } \\
\text { mixing }\end{array}$ & & $\begin{array}{l}\text { about } 94(1 \mathrm{C}, \\
500 \text { cycles, RT) }\end{array}$ & $\begin{array}{c}146\left(0.2 \mathrm{C}, 25^{\circ} \mathrm{C}\right) \\
106\left(2 \mathrm{C}, 25^{\circ} \mathrm{C}\right)\end{array}$ & 4 \\
\hline $\begin{array}{c}\text { MOF- } \\
525(\mathrm{Cu}) / \mathrm{PTFE}(9 \\
: 1)\end{array}$ & $\begin{array}{l}\text { Physical } \\
\text { mixing }\end{array}$ & & $\begin{array}{c}132(0.1 \mathrm{C}, 100 \\
\text { cycles, RT }) \\
\left(\mathrm{LiFePO}_{4}\right. \\
\text { loading of } \approx 25 \\
\left.\mathrm{mg} \mathrm{cm}^{-2}\right)\end{array}$ & $\begin{array}{c}68(0.05 \mathrm{C}, \\
\left.-20^{\circ} \mathrm{C}\right) \\
93\left(0.5 \mathrm{C}, 25^{\circ} \mathrm{C}\right) \\
143\left(0.5 \mathrm{C}, 80^{\circ} \mathrm{C}\right)\end{array}$ & 5 \\
\hline $\begin{array}{c}\text { M-UiO-66- } \\
\mathrm{NH}_{2} / \mathrm{PEGDA} \\
(1: 8)\end{array}$ & $\begin{array}{l}\text { photopol } \\
\text { ymerizati } \\
\text { on }\end{array}$ & $\begin{array}{c}2500 \Omega \\
(\mathrm{RT})\end{array}$ & $\begin{array}{c}130(0.2 \mathrm{C}, 100 \\
\text { cycles, } \sim 90 \% \\
\text { retention RT })\end{array}$ & $\begin{array}{c}151\left(0.2 \mathrm{C}, 60^{\circ} \mathrm{C}\right) \\
147\left(0.5 \mathrm{C}, 60^{\circ} \mathrm{C}\right) \\
74\left(1 \mathrm{C}, 25^{\circ} \mathrm{C}\right)\end{array}$ & 6 \\
\hline $\begin{array}{c}\text { ZIF-67/ILE } \\
(2: 3)\end{array}$ & $\begin{array}{l}\text { Physical } \\
\text { mixing }\end{array}$ & & $\begin{array}{l}151(0.1 \mathrm{C}, 60 \\
\left.\text { Cycles } 60^{\circ} \mathrm{C}\right)\end{array}$ & - & 8 \\
\hline $\begin{array}{c}\text { D-UiO-66- } \\
\mathrm{NH}_{2} / \mathrm{PEO} \\
(12.5 \text { vol\% D- } \\
\text { UiO-66-NH } 2 \text { ) }\end{array}$ & $\begin{array}{l}\text { Physical } \\
\text { mixing }\end{array}$ & $\begin{array}{c}324.1 \Omega \\
\left(60^{\circ} \mathrm{C}\right)\end{array}$ & $\begin{array}{c}\text { about } 91.5(1 \\
\text { C, } 60 \text { cycles, } \\
\left.60^{\circ} \mathrm{C}\right)\end{array}$ & $\begin{array}{c}126.4(0.5 \mathrm{C}, \\
\left.60{ }^{\circ} \mathrm{C}\right) \\
106.3\left(1 \mathrm{C}, 60^{\circ} \mathrm{C}\right) \\
67.4\left(5 \mathrm{C}, 60^{\circ} \mathrm{C}\right) \\
\end{array}$ & 9 \\
\hline $\begin{array}{c}\text { UiO-66@Li- } \\
\text { IL/PEO } \\
(4: 6)\end{array}$ & $\begin{array}{l}\text { Physical } \\
\text { mixing }\end{array}$ & & $\begin{array}{l}144(0.5 \mathrm{C}, 60 \\
\left.\text { cycles, } 60^{\circ} \mathrm{C}\right)\end{array}$ & 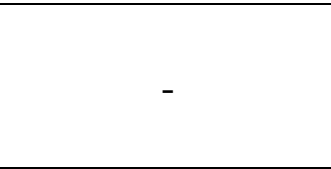 & 11 \\
\hline $\begin{array}{c}\text { MOF-688/PVDF } \\
(3: 1)\end{array}$ & $\begin{array}{c}\text { Physical } \\
\text { mixing }\end{array}$ & $\begin{array}{c}353 \Omega \\
(\mathrm{RT})\end{array}$ & $\begin{array}{c}120(\sim 0.2 \mathrm{C}, \\
200 \text { cycles, } \mathrm{RT})\end{array}$ & - & 12 \\
\hline $\begin{array}{c}\text { UiOLiTFSI/PVD } \\
\text { F } \\
(8: 2)\end{array}$ & $\begin{array}{l}\text { Physical } \\
\text { mixing }\end{array}$ & $\begin{array}{c}100 \Omega \\
(\mathrm{RT})\end{array}$ & $\begin{array}{c}132(1 \mathrm{C}, 500 \\
\text { Cycles RT }\end{array}$ & $\begin{array}{c}145\left(0.2 \mathrm{C}, 25^{\circ} \mathrm{C}\right) \\
136\left(1 \mathrm{C}, 25^{\circ} \mathrm{C}\right) \\
109\left(3 \mathrm{C}, 25^{\circ} \mathrm{C}\right) \\
98.6\left(1 \mathrm{C}, 0^{\circ} \mathrm{C}\right)\end{array}$ & 14 \\
\hline $\begin{array}{c}\text { NMS/PEO } \\
(8 \mathrm{wt} \% \text { NMS })\end{array}$ & $\begin{array}{l}\text { Physical } \\
\text { mixing }\end{array}$ & & $\begin{array}{l}125(0.1 \mathrm{C}, 50 \\
\left.\text { Cycles } 30^{\circ} \mathrm{C}\right)\end{array}$ & $\begin{array}{c}113\left(0.2 \mathrm{C}, 30^{\circ} \mathrm{C}\right) \\
62\left(0.5 \mathrm{C}, 30^{\circ} \mathrm{C}\right) \\
147\left(0.2 \mathrm{C}, 50^{\circ} \mathrm{C}\right) \\
132\left(1 \mathrm{C}, 50^{\circ} \mathrm{C}\right)\end{array}$ & 15 \\
\hline $\begin{array}{c}\text { UiO-66- } \\
\text { LiSS/PVDF } \\
(3: 1)\end{array}$ & $\begin{array}{l}\text { Physical } \\
\text { mixing }\end{array}$ & & $\begin{array}{c}112(0.2 \mathrm{C}, 100 \\
\text { Cycles RT) }\end{array}$ & $\begin{array}{c}127\left(0.2 \mathrm{C}, 25^{\circ} \mathrm{C}\right) \\
98\left(1 \mathrm{C}, 25^{\circ} \mathrm{C}\right) \\
88\left(2 \mathrm{C}, 25^{\circ} \mathrm{C}\right)\end{array}$ & 17 \\
\hline $\begin{array}{c}\text { ZIF-8/PEO- } \\
\text { LiTFSI }\end{array}$ & $\begin{array}{l}\text { Physical } \\
\text { mixing }\end{array}$ & & $\begin{array}{c}111(0.5 \mathrm{C}, 350 \\
\left.\text { cycles, } 60^{\circ} \mathrm{C}\right)\end{array}$ & $\begin{array}{l}132\left(0.2 \mathrm{C}, 60^{\circ} \mathrm{C}\right) \\
126\left(0.5 \mathrm{C}, 60^{\circ} \mathrm{C}\right)\end{array}$ & 18 \\
\hline
\end{tabular}




\begin{tabular}{|c|c|c|c|c|c|}
\hline$(15 \%: 85 \%)$ & & & & $109\left(1 \mathrm{C}, 60^{\circ} \mathrm{C}\right)$ & \\
\hline $\begin{array}{c}\text { Li-IL@UiO- } \\
\text { 67/PVDF-HFP } \\
(1.8: 1)\end{array}$ & $\begin{array}{l}\text { Physical } \\
\text { mixing }\end{array}$ & & $\begin{array}{c}140(0.1 \text { C, } 300 \\
\text { Cycles RT }) \\
150(0.3 \text { C, } 140 \\
\text { Cycles, RT })\end{array}$ & $\begin{array}{c}137.1(0.5 \mathrm{C}, \mathrm{RT}) \\
118.1(1 \mathrm{C}, \mathrm{RT}) \\
72.4(3 \mathrm{C}, \mathrm{RT})\end{array}$ & 19 \\
\hline $\begin{array}{l}\text { LCMOF- } \\
\text { 1/PVDF } \\
(7: 3)\end{array}$ & $\begin{array}{l}\text { Physical } \\
\text { mixing }\end{array}$ & & $\begin{array}{l}150(1 \mathrm{C}, 500 \\
\left.\text { Cycles, } 60^{\circ} \mathrm{C}\right)\end{array}$ & $\begin{array}{c}100(0.05 \mathrm{C}, \\
\left.-20^{\circ} \mathrm{C}\right) \\
135\left(1 \mathrm{C}, 30^{\circ} \mathrm{C}\right)\end{array}$ & 20 \\
\hline $\begin{array}{c}\text { Li-IL@HKUST- } \\
1 / \mathrm{PEO} \\
(3: 2)\end{array}$ & $\begin{array}{l}\text { Physical } \\
\text { mixing }\end{array}$ & & $\begin{array}{c}136.2(1 \mathrm{C}, 100 \\
\left.\text { Cycles, } 25^{\circ} \mathrm{C}\right)\end{array}$ & $\begin{array}{c}150.6(0.2 \mathrm{C}, \\
\left.25^{\circ} \mathrm{C}\right) \\
141.6\left(1 \mathrm{C}, 25^{\circ} \mathrm{C}\right) \\
134.4\left(2 \mathrm{C}, 25^{\circ} \mathrm{C}\right) \\
\end{array}$ & 21 \\
\hline $\begin{array}{l}\text { ZIF-8@Li- } \\
\text { IL/PEO } \\
(6: 4)\end{array}$ & $\begin{array}{l}\text { Physical } \\
\text { mixing }\end{array}$ & $\begin{array}{c}132 \Omega \\
\left(60^{\circ} \mathrm{C}\right)\end{array}$ & $\begin{array}{c}\text { about } 122.88 \\
(0.1 \mathrm{C}, 120 \\
\text { Cycles, RT })\end{array}$ & $\begin{array}{l}160(0.1 \mathrm{C}, \mathrm{RT}) \\
130(0.2 \mathrm{C}, \mathrm{RT}) \\
105(0.5 \mathrm{C}, \mathrm{RT})\end{array}$ & 22 \\
\hline $\begin{array}{l}\mathrm{UiO} / \mathrm{Li}-\mathrm{IL} \\
(15: 16)\end{array}$ & $\begin{array}{l}\text { Physical } \\
\text { mixing }\end{array}$ & & $\begin{array}{c}130(0.2 \mathrm{C}, 100 \\
\left.\text { Cycles } 60^{\circ} \mathrm{C}\right) \\
119(1 \mathrm{C}, 380 \\
\left.\text { Cycles, } 60^{\circ} \mathrm{C}\right)\end{array}$ & & 23 \\
\hline $\begin{array}{c}\text { ZIF- } \\
\text { 8/PEO=10:1; } \\
\text { PEGDA/BMA=2 } \\
: 1\end{array}$ & $\begin{array}{c}\text { Physical } \\
\text { mixing/ } \\
\text { thermal- } \\
\text { initiated } \\
\text { polymeri } \\
\text { zation }\end{array}$ & $\begin{array}{c}130 \Omega \\
(\mathrm{RT})\end{array}$ & $\begin{array}{c}110(1 \mathrm{C}, 300 \\
\text { Cycles, RT) }\end{array}$ & $\begin{array}{c}159 \text { (0.2 C, RT) } \\
120(1 \mathrm{C}, \mathrm{RT}) \\
60(5 \mathrm{C}, \mathrm{RT})\end{array}$ & 24 \\
\hline $\mathrm{MOF}=64 \%$ & $\begin{array}{l}\text { Physical } \\
\text { mixing }\end{array}$ & & & $\begin{array}{c}150(0.2 \mathrm{C}, \mathrm{RT}) \\
120(1 \mathrm{C}, \mathrm{RT})\end{array}$ & 25 \\
\hline $\begin{array}{l}\mathrm{Zr}-\mathrm{BPDC}- \\
2 \mathrm{SO}_{3} \mathrm{H} / \mathrm{BC} \\
\quad(7: 3)\end{array}$ & $\begin{array}{l}\text { In situ } \\
\text { growth }\end{array}$ & $\begin{array}{l}74 \Omega \\
(\mathrm{RT})\end{array}$ & $\begin{array}{c}140(1 \mathrm{C}, 500 \\
\text { Cycles RT }) \\
119(3 \mathrm{C}, 600 \\
\text { Cycles, RT })\end{array}$ & $\begin{array}{c}160 \text { (0.2 C, RT) } \\
143 \text { (1 C, RT) } \\
108 \text { (5 C, RT) }\end{array}$ & $\begin{array}{l}\text { This } \\
\text { work }\end{array}$ \\
\hline
\end{tabular}




\section{References}

(1) Wang, H.; Zhou, L.; Wang, Y.; Liu, Q. Terbium-biphenyl-3,3'-disulfonyl-4,4'dicarboxylate framework with sulfonate sites for luminescent sensing of $\mathrm{Cr}^{3+}$ ion. Inorg. Chem. Commun. 2016, 73, 94.

(2) Wiers, B. M.; Foo, M. L.; Balsara, N. P.; Long, J. R. A solid lithium electrolyte via addition of lithium isopropoxide to a metal-organic framework with open metal sites. J. Am. Chem. Soc. 2011, 133, 14522.

(3) Park, S. S.; Tulchinsky, Y.; Dinca, M. Single-ion $\mathrm{Li}^{+}, \mathrm{Na}^{+}$, and $\mathrm{Mg}^{2+}$ solid electrolytes supported by a mesoporous anionic $\mathrm{Cu}$-azolate metal-organic framework. J. Am. Chem. Soc. 2017, 139, 13260.

(4) Shen, L.; Wu, H. B.; Liu, F.; Brosmer, J. L.; Shen, G.; Wang, X.; Zink, J. I.; Xiao, Q.; Cai, M.; Wang, G.; Lu, Y.; Dunn, B. Creating lithium-ion electrolytes with biomimetic ionic channels in metal-organic frameworks. Adv. Mater. 2018, 30, 1707476.

(5) Wang, Z.; Tan, R.; Wang, H.; Yang, L.; Hu, J.; Chen, H.; Pan, F. A metal-organicframework-based electrolyte with nanowetted interfaces for high-energy-density solid-state lithium battery. Adv. Mater. 2018, 30, 1704436.

(6) Wang, Z.; Wang, S.; Wang, A.; Liu, X.; Chen, J.; Zeng, Q.; Zhang, L.; Liu, W.; Zhang, L. Covalently linked metal-organic framework (MOF)-polymer all-solidstate electrolyte membranes for room temperature high performance lithium 
batteries. J. Mater. Chem. A 2018, 6, 17227.

(7) Wang, Z.; Wang, Z.; Yang, L.; Wang, H.; Song, Y.; Han, L.; Yang, K.; Hu, J.; Chen, H.; Pan, F. Boosting interfacial $\mathrm{Li}^{+}$transport with a MOF-based ionic conductor for solid-state batteries. Nano Energy 2018, 49, 580.

(8) Chen, N.; Li, Y.; Dai, Y.; Qu, W.; Xing, Y.; Ye, Y.; Wen, Z.; Guo, C.; Wu, F.; Chen, $\mathrm{R} . \mathrm{A} \mathrm{Li}^{+}$conductive metal organic framework electrolyte boosts the hightemperature performance of dendrite-free lithium batteries. J. Mater. Chem. A 2019, 7, 9530 .

(9) Huo, H.; Wu, B.; Zhang, T.; Zheng, X.; Ge, L.; Xu, T.; Guo, X.; Sun, X. Anionimmobilized polymer electrolyte achieved by cationic metal-organic framework filler for dendrite-free solid-state batteries. Energy Storage Mater. 2019, 18, 59.

(10) Miner, E. M.; Park, S. S.; Dinca, M. High $\mathrm{Li}^{+}$and $\mathrm{Mg}^{2+}$ conductivity in a $\mathrm{Cu}-$ azolate metal-organic framework. J. Am. Chem. Soc. 2019, 141, 4422.

(11) Wu, J.; Guo, X. MOF-derived nanoporous multifunctional fillers enhancing the performances of polymer electrolytes for solid-state lithium batteries. J. Mater. Chem. A 2019, 7, 2653.

(12) Xu, W.; Pei, X.; Diercks, C. S.; Lyu, H.; Ji, Z.; Yaghi, O. M. A metal-organic framework of organic vertices and polyoxometalate linkers as a solid-state electrolyte. J. Am. Chem. Soc. 2019, 141, 17522.

(13) Sun, C.; Zhang, J. H.; Yuan, X. F.; Duan, J. N.; Deng, S. W.; Fan, J. M.; Chang, 
J. K.; Zheng, M. S.; Dong, Q. F. ZIF-8-based quasi-solid-state electrolyte for lithium batteries. ACS Appl. Mater. Interfaces 2019, 11, 46671.

(14) Zhu, F.; Bao, H.; Wu, X.; Tao, Y.; Qin, C.; Su, Z.; Kang, Z. High-performance metal-organic framework-based single ion conducting solid-state electrolytes for low-temperature lithium metal batteries. ACS Appl. Mater. Interfaces 2019, 11, 43206.

(15) Han, Q.; Wang, S.; Jiang, Z.; Hu, X.; Wang, H. Composite polymer electrolyte incorporating metal-organic framework nanosheets with improved electrochemical stability for all-solid-state Li metal batteries. ACS Appl. Mater. Interfaces 2020, 12, 20514.

(16) Angulakshmi, N.; Zhou, Y.; Suriyakumar, S.; Dhanalakshmi, R. B.; Satishrajan, M.; Alwarappan, S.; Alkordi, M. H.; Stephan, A. M. Microporous metal-organic framework (MOF)-based composite polymer electrolyte (CPE) mitigating lithium dendrite formation in all-solid-state-lithium batteries. ACS Omega 2020, 5, 7885.

(17) Yang, H.; Liu, B.; Bright, J.; Kasani, S.; Yang, J.; Zhang, X.; Wu, N. A singleIon conducting UiO-66 metal-organic framework electrolyte for all-solid-state lithium batteries. ACS Appl. Energy Mater. 2020, 3, 4007.

(18) Lei, Z.; Shen, J.; Zhang, W.; Wang, Q.; Wang, J.; Deng, Y.; Wang, C. Exploring porous (zeolitic imidazolate frame work-8) ZIF-8 as an efficient filler for highperformance poly(ethyleneoxide)-based solid polymer electrolytes. Nano Res. 
2020, 13, 2259.

(19) Liu, L.; Sun, C. Flexible quasi-solid-state composite electrolyte membrane derived from a metal-organic framework for lithium-metal batteries. ChemElectroChem 2020, 7, 707.

(20) Zhang, Q.; Li, D.; Wang, J.; Guo, S.; Zhang, W.; Chen, D.; Li, Q.; Rui, X.; Gan, L.; Huang, S. Multiscale optimization of Li-ion diffusion in solid lithium metal battery via ion conductive metal-organic frameworks. Nanoscale 2020, 12, 6976.

(21) Wang, Z.; Zhou, H.; Meng, C.; Xiong, W.; Cai, Y.; Hu, P.; Pang, H.; Yuan, A. Enhancing ion transport: function of ionic liquid decorated MOFs in polymer electrolytes for all-solid-state lithium batteries. ACS Appl. Energy Mater. 2020, 3, 4265 .

(22) Xia, Y.; Xu, N.; Du, L.; Cheng, Y.; Lei, S.; Li, S.; Liao, X.; Shi, W.; Xu, L.; Mai, L. Rational design of ion transport paths at the interface of metal-organic framework modified solid electrolyte. ACS Appl. Mater. Interfaces 2020, 12, 22930.

(23) Wu, J. F.; Guo, X. Nanostructured metal-organic framework (MOF)-derived solid electrolytes realizing fast lithium ion transportation kinetics in solid-state batteries. Small 2019, 15, 1804413.

(24) Wang, G; He, P; Fan, L. Asymmetric polymer electrolyte constructed by metalorganic framework for solid-state, dendrite-free lithium metal battery. Adv. Funct. 
Mater. 2021, 31, 2007198.

(25) Sun, J; Yao, X; Li, Y; Zhang, Q; Hou C; Shi, Q; Wang H.. Facilitating interfacial stability via bilayer heterostructure solid electrolyte toward high-energy, safe and adaptable lithium batteries. Adv. Energy Mater. 2020, 2000709. 